\title{
Laser polishing of niobium for superconducting radio-frequency accelerator applications
}

\author{
Liang Zhao, ${ }^{1,2}$ J. Michael Klopf, ${ }^{1}$ Charles E. Reece, ${ }^{2}$ and Michael J. Kelley ${ }^{1,2, *}$ \\ ${ }^{1}$ Applied Science Department, The College of William and Mary, Williamsburg, Virginia 23187, USA \\ ${ }^{2}$ Thomas Jefferson National Accelerator Facility, Newport News, Virginia 23606, USA
}

(Received 19 November 2013; published 26 August 2014)

\begin{abstract}
Interior surfaces of niobium cavities used in superconducting radio frequency accelerators are now obtained by buffered chemical polish and/or electropolish. Laser polishing is a potential alternative, having advantages of speed, freedom from noxious chemistry and availability of in-process inspection. We studied the influence of the laser power density and laser beam raster rate on the surface topography. These two factors need to be combined carefully to smooth the surface without damage. Computational modeling was used to estimate the surface temperature and gain insight into the mechanism of laser polishing. Power spectral density analysis of surface topography measurements shows that laser polishing can produce smooth topography similar to that obtained by electropolish. This is a necessary first step toward introducing laser polishing as an alternative to the currently practiced chemical polishing.
\end{abstract}

DOI: 10.1103/PhysRevSTAB.17.083502

PACS numbers: 81.05.-t, 81.65.-b

\section{INTRODUCTION}

The final step in the production of niobium superconducting radio-frequency (SRF) accelerator cavities is removal of $\sim 100 \mu \mathrm{m}$ from the interior surface [1] by chemical and/or mechanical methods in order to remove contaminants and damage. Buffered chemical polishing (BCP), which provides a niobium surface etch with a mixture of hydrofluoric (HF), nitric acid and phosphoric acids has long been the dominant process because of simplicity and effectiveness. Electropolishing (EP) with a hydrofluoric-sulfuric acid mixture sees increasing use as a final step to remove performance-limiting [2,3] sharp features caused by BCP, but suffers a slower etching rate and uses a more complex setup [4]. Both involve hazardous acids and have potential for hydrogen contamination problems. Therefore, pursuit of greener and/or faster treatment methods has never stopped. Centrifugal barrel polishing (CBP), a mechanical method, avoids aggressive chemical and provides mirrorlike surface, but the price is much longer polishing time. CBP may use a light EP as a final step [5,6]. Other approaches include non-HF EP [7-11], plasma etching [12,13] and laser polishing [14,15].

Laser polishing relies on beam heating to partially melt the metal surface. Partial melting (or shallow surface melting, SSM) is preferred over formation of a full liquid surface layer (or surface overlayer melting, SOM) [16].

\footnotetext{
*Corresponding author. mkelley@jlab.org

Published by the American Physical Society under the terms of the Creative Commons Attribution 3.0 License. Further distribution of this work must maintain attribution to the author $(s)$ and the published article's title, journal citation, and DOI.
}

The absence of a fully formed surface melt layer avoids hydrodynamical effects such as the formation of ripples that can remain upon solidification, a potentially harmful sharp roughness. Usually pulsed lasers are used so that the melt duration is short and cooling happens quickly. During the short melt period (a few nanoseconds), the surface tension works to smooth out sharp features. The final smoothening is the consequence of the total melt duration, the sum of all pulses delivered to a location. Since the effect of any pulse is reduced by the cumulative smoothening resulting from previous pulses, it is expected that there will be an optimum total melt time (i.e., number of laser pulses) for cost effectiveness.

Laser polishing has received researchers' attention for years, and significant experience has been gained. Examples include using $\mathrm{CO}_{2}$ lasers and diode lasers to polish tool steel by removing surface patterns from machining [17]. Pulsed laser micropolishing $(\mathrm{PL} \mu \mathrm{P})$ as an alternative to chemical mechanical polishing on nickel [18] not only reduced surface roughness but also increased corrosion resistance, as was also found on stainless steel after laser polishing [19]. Titanium medical implant material revealed a decrease of surface contamination and thickening of oxide layer after laser polishing [20]. Laser polishing is also applied to optical glass [21]. Previously we found that laser polishing reduced the rms surface roughness of mechanically polished only niobium from $700 \mathrm{~nm}$ down to $200 \mathrm{~nm}$ [14]. Within the fluence range of $0.6-2.3 \mathrm{~J} / \mathrm{cm}^{2}$ and $80-900$ pulses overlapping, both SMM and SOM contributed to the resulting surface. This shows laser polishing of the niobium SRF cavity is promising and that there is opportunity for further improvement as well. Later we found that laser polishing can achieve still smoother topography, similar to that obtained by EP [15]. 
The present study aims at exploring in detail a wider range of polishing parameters, seeking even better surface finishing. The parameters to be optimized are fluence (energy per unit area per pulse) and pulse displacement. Pulse displacement is defined as the distance between the centers of two consecutive pulses. The product of pulse displacement and number of pulses overlapped equals one spot width. This is actually a measure of total energy deposition density onto the metal surface.

\section{SIMULATION}

Before undertaking experiments, it is desirable to obtain a sense of the range of key parameters and their effects. We previously found that a straightforward single pulse analysis provides insight. It is not the purpose of such a model to accurately represent the complete process in detail, but rather to guide the experiments. In that regard, a useful benchmark is the energy requirement to bring niobium surface into the SSM melt range in a single pulse.

Here a simplified model was used to simulate laser polishing as described in [22]. A one-dimensional conduction heat transfer equation [Eq. (1)]

$$
\frac{\partial T(z, t)}{\partial t}-\frac{\partial}{\partial t}\left(k(T) \frac{\partial T(z, t)}{\partial t}\right)=\frac{A(z, t)}{\rho(T) c_{p}(T)}
$$

is used to model the temperature at the center of the laser spot, where $T(z, t)$ is the temperature at depth $z$ at time $t, \rho$ is the mass density, $k=k^{\prime} / \rho c_{p}$ is the thermal diffusivity, $k^{\prime}$ is the specific thermal conductivity, and $c_{p}$ is the specific heat capacity. The material is treated as a one-dimensional semi-infinite solid, because the diameter of the melted zone is large compared to the melt depth ( $\sim 100 \mu \mathrm{m}$ vs $\sim 100 \mathrm{~nm}$ respectively). The source term,

$$
A(z, t)=\alpha I(t)(1-R) e^{-\alpha z}+\Delta U(z, t),
$$

includes heat generated by absorption of the Gaussian laser pulse $\left[I(t)=I_{o} e^{-\left(t-t_{o}\right)^{2} / 2 \sigma^{2}}\right]$, where $\alpha$ is the optical absorption coefficient, and $R$ is the optical reflectivity at the laser wavelength. In Eq. (2), $\Delta U(z, t)$ represents the internal heat sinks associated with the latent heat of the phase transformations of the material. Cooling is by conduction only; no radiant or convective heat loss was considered. The material is homogeneous and the density, $\rho$, is taken to be constant, but temperature dependent values for $k^{\prime}$ and $c_{p}$ are used in the finite difference method applied to numerically solving Eq. (1). While this method provides an excellent tool for simulating the temperature profile produced by absorption of a laser pulse, the wide range of local surface topography may have an even larger impact on the actual temperature profile realized on real $\mathrm{Nb}$ samples.

A plot of the simulated surface temperature is shown in Fig. 1. The dashed line corresponds to the melting temperature and the dot-dashed to boiling. The figure shows the time course of surface temperature associated a single $8 \mathrm{~ns}$ laser pulse for the indicated fluences, and the heat sink due to the latent heat of melting is apparent, particularly during cooling.

The simulation also computes the temperature vs depth profile and the maximum depth of melting which can play an important role in the resulting laser polished surfaces. The maximum melt depth is $375 \mathrm{~nm}$ for a fluence of $2.75 \mathrm{~J} / \mathrm{cm}^{2}, 127 \mathrm{~nm}$ for $2.25 \mathrm{~J} / \mathrm{cm}^{2}$, and $60 \mathrm{~nm}$ for $2.0 \mathrm{~J} / \mathrm{cm}^{2}$. We note that these conditions allow for

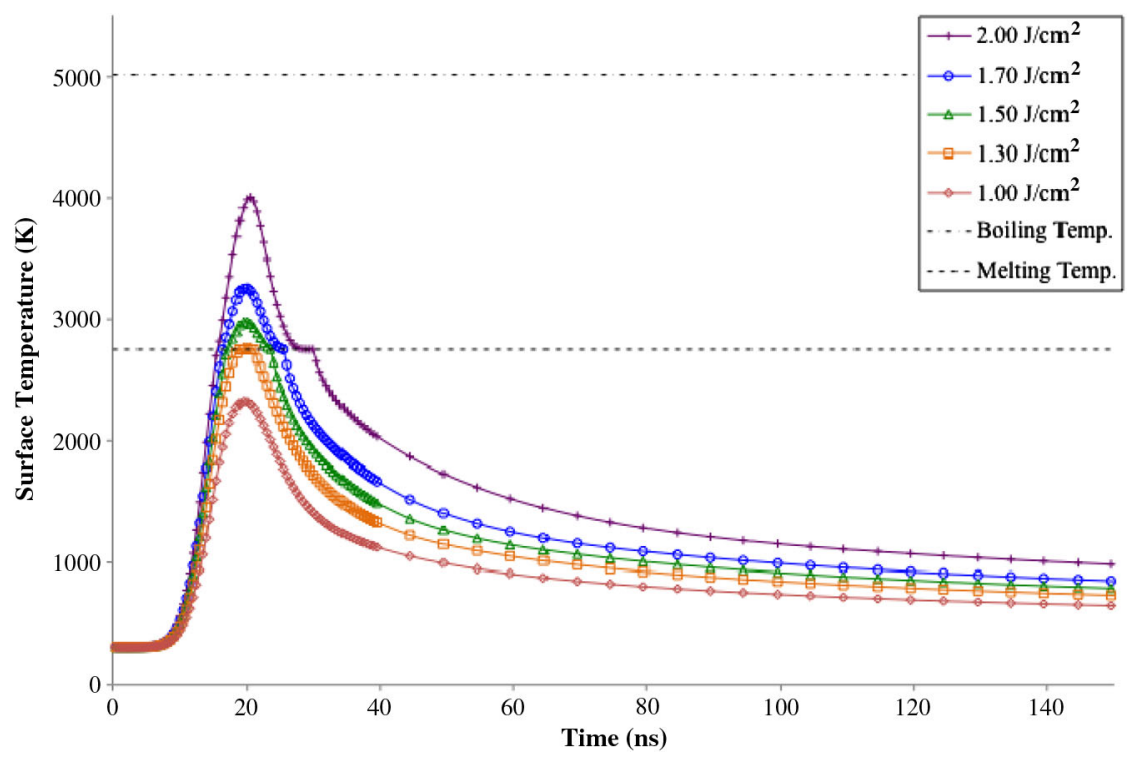

FIG. 1. The simulated surface temperature for $\mathrm{Nb}$ due to the absorption of a $8 \mathrm{~ns} 1064 \mathrm{~nm}$ laser pulse for varying fluence values. The melting and boiling temperature are shown as dashed and dot-dashed lines respectively. 
recrystallization of the superconducting rf penetration depth $(\sim 40 \mathrm{~nm})$.

The accumulation of residual heat when multiple laser pulses are incident on the surface has also been simulated, and the results indicate a small sequential rise in the peak temperature and melt depth for each laser pulse. On the other hand, at the fluence conditions for laser polishing, the reflectance of the real $\mathrm{Nb}$ surface will improve sequentially with each laser pulse, resulting in falling absorption (heating) with each pulse. This effect counters the residual heat accumulation, helping to regulate the peak temperature and melt depth over the treatment of many laser pulses.

To summarize, the expected sequence of events is that a pulse arrives at an ambient temperature surface. The surface temperature rises until the end of the pulse, followed by temperatures below the surface. Accumulating heat from previous pulses to the same location will move the fluence requirement for both these temperatures lower. Differences of surface topography arising from different prior processing history can affect absorption of the laser beam energy, leading to differences in heating. Further, each successive pulse will bring a corresponding increment of smoothening. This will change both the energy absorbed from the beam and reduce the extent of further smoothening during the melt duration from the present pulse. Uncertainties aside, this simple simulation serves to direct attention to the range of a few-tenths $\mathrm{J} / \mathrm{cm}^{2}$ per pulse, with details to be resolved by experiment.

\section{EXPERIMENTAL METHODS}

\section{A. Laser treatment system}

A spectra-physics high intensity peak power oscillator tabletop laser, optimized for micromachining, operating at $1064 \mathrm{~nm}$ was used as the laser source. The beam was measured to be a Gaussian with a Spiricon CCD camera based beam profiler. The beam was directed into the UHV chamber of a PLD-5000 System from PVD Products Inc. The nominal angle of incidence of the laser beam on sample was $60^{\circ}$. The focused spot size on the sample was $62 \mu \mathrm{m} \times$ $124 \mu \mathrm{m}$ as measured by a knife-edge technique at the sample surface. The beam intensity at the sample position was measured as the change in reflected intensity when traversing a razor blade across a high reflectivity sample. The repetition rate was $19 \mathrm{kHz}$, and the pulse length at this repetition rate was about 8 ns. The sample holder was located at the center of the UHV chamber, as shown in Fig. 2, and each sample could rotate individually with a speed up to $60 \mathrm{rpm}$. During polishing, the sample rotating speed was adjusted accordingly to satisfy the pulse accumulation requirement. The chamber was pumped down to $\sim 10^{-7}-10^{-8}$ Torr before the polishing experiments. The pressure during polishing never exceeded the high $10^{-7}$ Torr range.

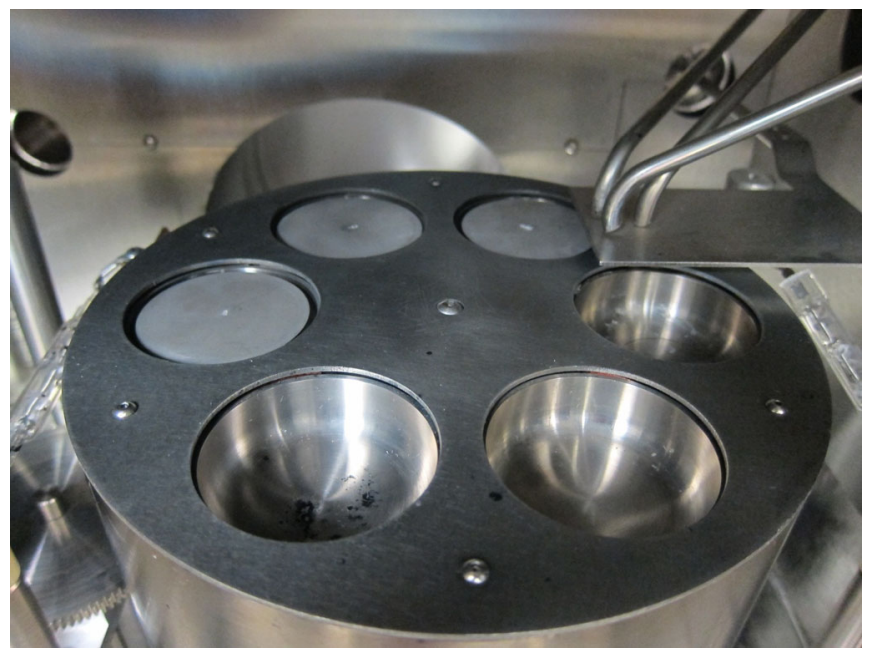

FIG. 2. The sample holder in the vacuum chamber of the laser treatment system.

\section{B. Material and preparation}

The niobium samples were $49 \mathrm{~mm}$ diameter disks (Fig. 3) cut from $3.2 \mathrm{~mm}$ thick sheet material of the type used for cavity fabrication. BCP practice varies in etch time and conditions depending on the application. We previously [24-26] reported that even a few microns removal by $\mathrm{BCP}$ produces the same topography as heavy removal, as revealed by power spectral density (PSD) analysis of atomic force microscopy data (see later). To enable multiple etch/polish cycles with the same samples, we employed a light etch, one minute in $1: 1: 1 \mathrm{BCP}$ solution at $23^{\circ} \mathrm{C}$, rinsed with deionized water and air dried. This procedure is used at Jefferson Lab to prepare cavities for welding. The sample disks were then loaded into the laser treatment system and pumped down.

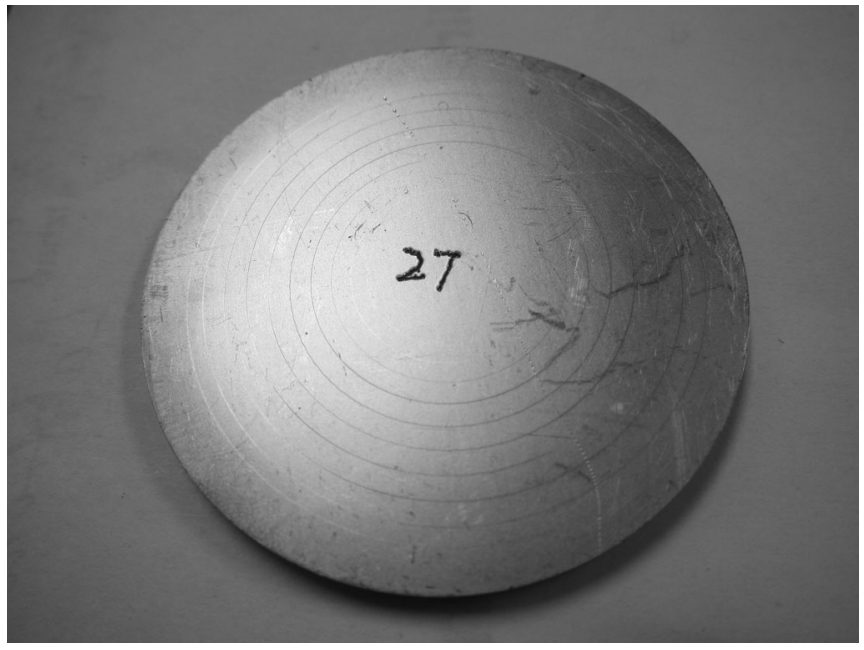

FIG. 3. A niobium sample disk with laser treated rings. 
TABLE I. Laser irradiation parameters.

\begin{tabular}{lcccc}
\hline \hline Laser power & Fluence & Number of pulses overlapped & Pulse displacement & Scan speed \\
\hline $\mathrm{W}$ & $\mathrm{J} / \mathrm{cm}^{2}$ & Within one beam width & $\mu \mathrm{m}$ & $\mathrm{cm} / \mathrm{s}$ \\
$1.51-10.98$ & $0.68-4.96$ & $10-670$ & $6.2-0.14$ & $11.78-0.27$ \\
\hline \hline
\end{tabular}

\section{Polishing parameters}

The fluence and the pulse accumulation were the two main parameters studied. The fluence was the energy density per pulse. The pulse accumulation could be expressed as the number of pulses overlapped within one spot width, or pulse displacement. During laser polishing the sample rotated about its own center at the chosen speed, while the beam was kept stationary or traversing along a radius. The target rotation was adjusted according to pulse accumulation required. With a stationary laser beam, each revolution of the target produced a polished single circle track on the sample. Changing the beam location on the sample yielded a series of concentric circles on the disks. Single track experiments were used for parameter optimization; Table I summarizes the range of these parameters explored. With laser beam moving along the radius of the sample in a series of steps, a ring comprised of multiple tracks could be produced. This experiment was used to explore the feasibility of treating large areas by beam rastering. The overlap fraction between tracks was explored from 0 to $100 \%$, seeking the smallest overlap that gave no visible seam.

\section{Characterization}

After laser treatment, the topography was examined by optical microscopy (HIROX KH-7700 High Resolution Digital-Video Microscopy System) and atomic force microscopy (AFM) (Digital Instruments Nanoscope IV). AFM was done in tapping mode with silicon tips and the scan size was $25 \mu \mathrm{m} \times 25 \mu \mathrm{m}$ or $50 \mu \mathrm{m} \times 50 \mu \mathrm{m}$ for each image. Each AFM image consisted of 512 scan lines and there were 512 data points for each line. Root mean square

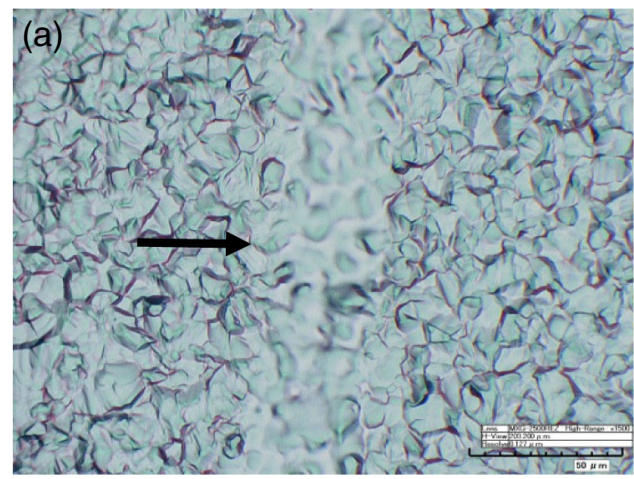

(rms) roughness values were obtained from these AFM data.

As we have discussed at length previously [26], roughness values are not a sufficiently incisive view of topography for the present purpose. The optics community encountered this issue long ago and turned to Fourier analysis to provide a direct view of the contributions at different length scales, power spectral density (PSD). PSD routines are found in the software packages accompanying AFM's, but they do not deal with a number of issues. It was therefore necessary for us to develop our own software, described elsewhere [24]. The understanding of detailed analysis of PSD data from SRF cavities is still advancing, but some inferences can reliably be made. As noted by optics researchers, sharp features lead to a straight line on a $\log / \log$ plot. Rounding the sharp edges leads to relatively greater intensity loss at short wavelengths. The topography arising from certain processes (BCP, EP) gives rise to characteristic PSD plots, useful as fingerprints, while development of a more fundamental understanding proceeds.

\section{RESULTS AND DISCUSSION}

We studied the effect of varying fluence at a fixed pulse accumulation, where 34 pulses overlapped within one spot width. We also studied the effect of varying pulse accumulation at a fixed fluence at $2.27 \mathrm{~J} / \mathrm{cm}^{2}$. These values were chosen based on the simulation discussed earlier.

Figure 4 shows optical images after laser treatment with two combinations of fluence and pulse accumulation: (A) $0.90 \mathrm{~J} / \mathrm{cm}^{2}, 34$ pulses overlapped; (B) $2.27 \mathrm{~J} / \mathrm{cm}^{2}$, 23 pulses overlapped. Both $0.90 \mathrm{~J} / \mathrm{cm}^{2}$ and $2.27 \mathrm{~J} / \mathrm{cm}^{2}$

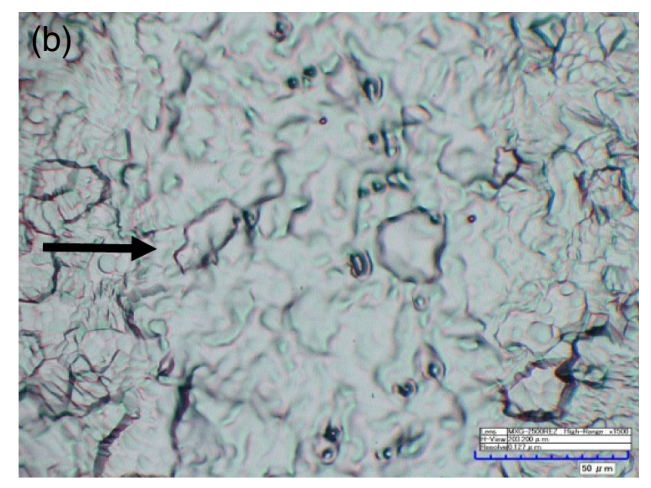

FIG. 4. Optical images of niobium surfaces after laser treatment: (a) $0.90 \mathrm{~J} / \mathrm{cm}^{2}, 34$ pulses overlapped, $1.8 \mu \mathrm{m}$ pulse displacement, scanning speed $3.5 \mathrm{~cm} / \mathrm{s}$. (b) $2.27 \mathrm{~J} / \mathrm{cm}^{2}, 23$ pulses overlapped, $3.0 \mu \mathrm{m}$ pulse displacement, scanning speed $5.6 \mathrm{~cm} / \mathrm{s}$. The arrows indicate the laser tracks. 

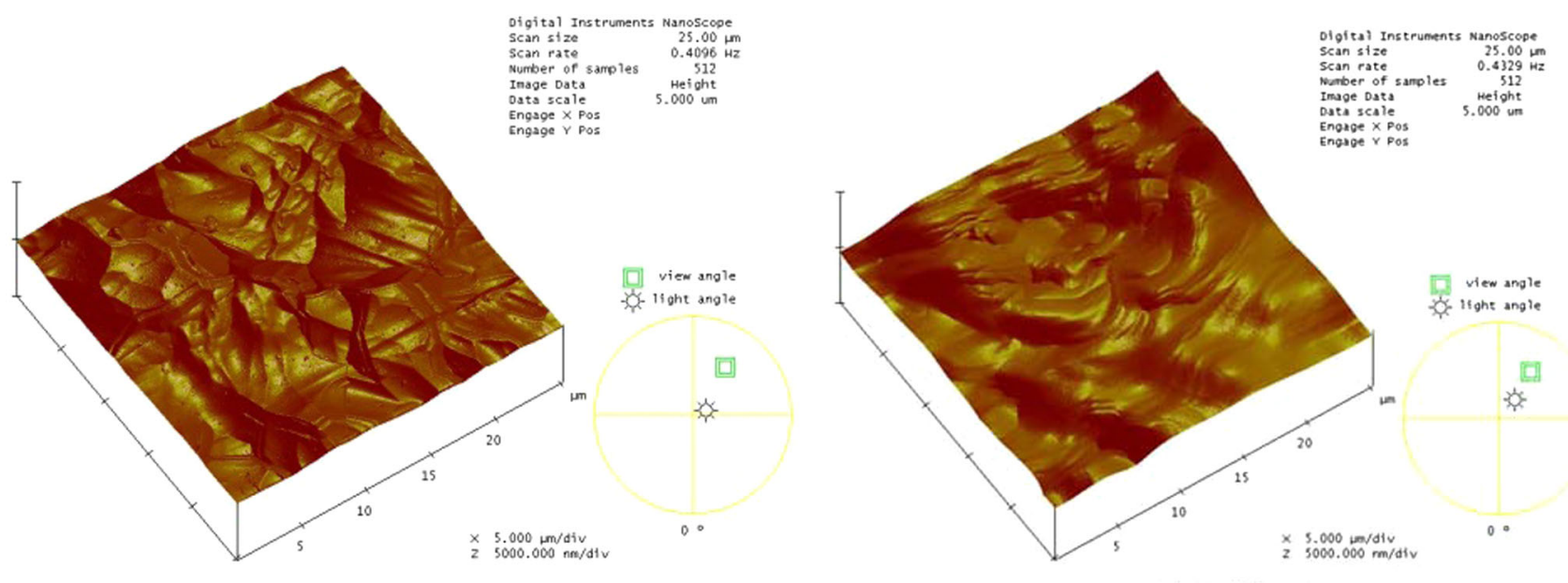

samp le-27-center-25um. 000
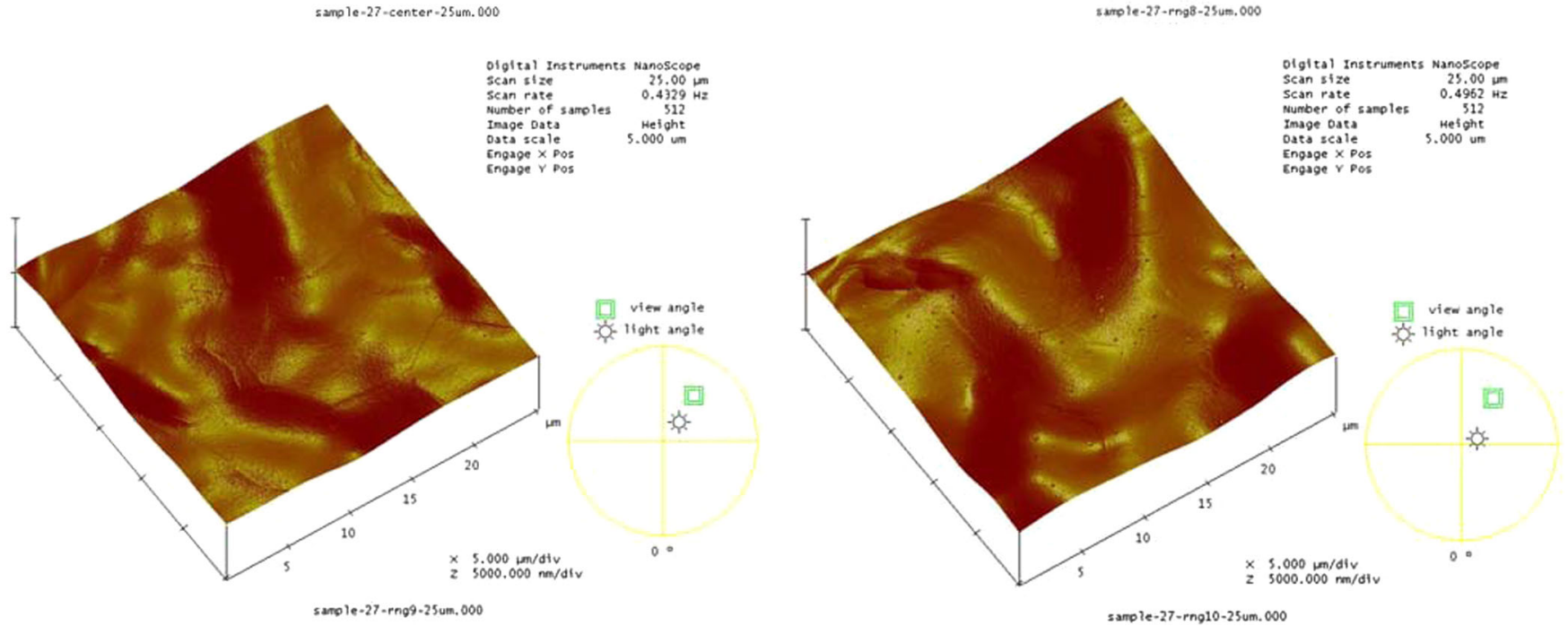

FIG. 5. AFM images of the niobium surface after laser treatment, with 34 pulses overlapped and fluence indicated. Top left, BCP surface without laser treatment; top right, $1.13 \mathrm{~J} / \mathrm{cm}^{2}$; lower left, $0.90 \mathrm{~J} / \mathrm{cm}^{2}$; lower right, $0.68 \mathrm{~J} / \mathrm{cm}^{2}$. Scan area $25 \mu \mathrm{m} \times 25 \mu \mathrm{m}$. Horizontal scale: $5 \mu \mathrm{m}$ per division. Vertical scale: $0.5 \mu \mathrm{m}$ per division.

cases showed a smoothened surface. Notice that the scanning speed was 3.5 and $5.6 \mathrm{~cm} / \mathrm{s}$, respectively. If the energy per pulse is high, then fewer pulses are needed in a given area accordingly, enabling a higher polishing rate. Here, a fluence of $0.90 \mathrm{~J} / \mathrm{cm}^{2}$ with 34 pulses overlapping provided the smoothest surface.

The next two figures view the effect of varying fluence at constant pulse accumulation. Figure 5 shows the AFM images of material treated at 0 (top left), 1.13 (top right), 0.90 (bottom left) or $0.68 \mathrm{~J} / \mathrm{cm}^{2}$ (lower right) fluence, with 34 pulses overlapped. The top left image is a typical BCP surface. The crater and the sharp edges are signatures of chemical etching. The top right is a slightly over melted surface, where ripples pile up resulting in a rough surface. Both lower images are both polished surfaces, where the sharp edges from BCP are smoothed but no new roughening is introduced.

Figure 6 presents the PSD analysis corresponding to the AFM images in Fig. 5. The BCP treated surface shows the

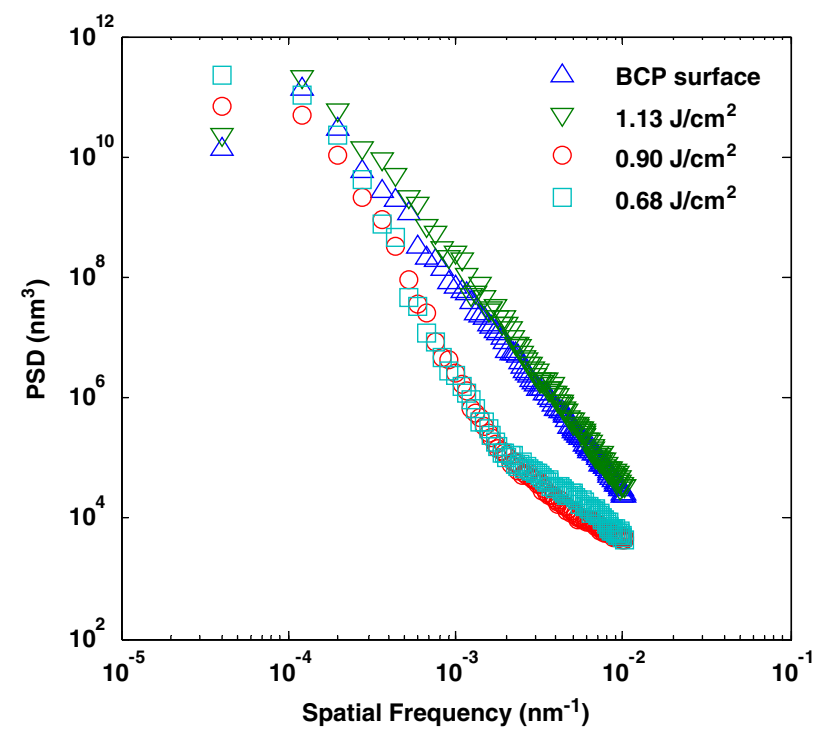

FIG. 6. PSD of the AFM data corresponding to Fig. 5. 

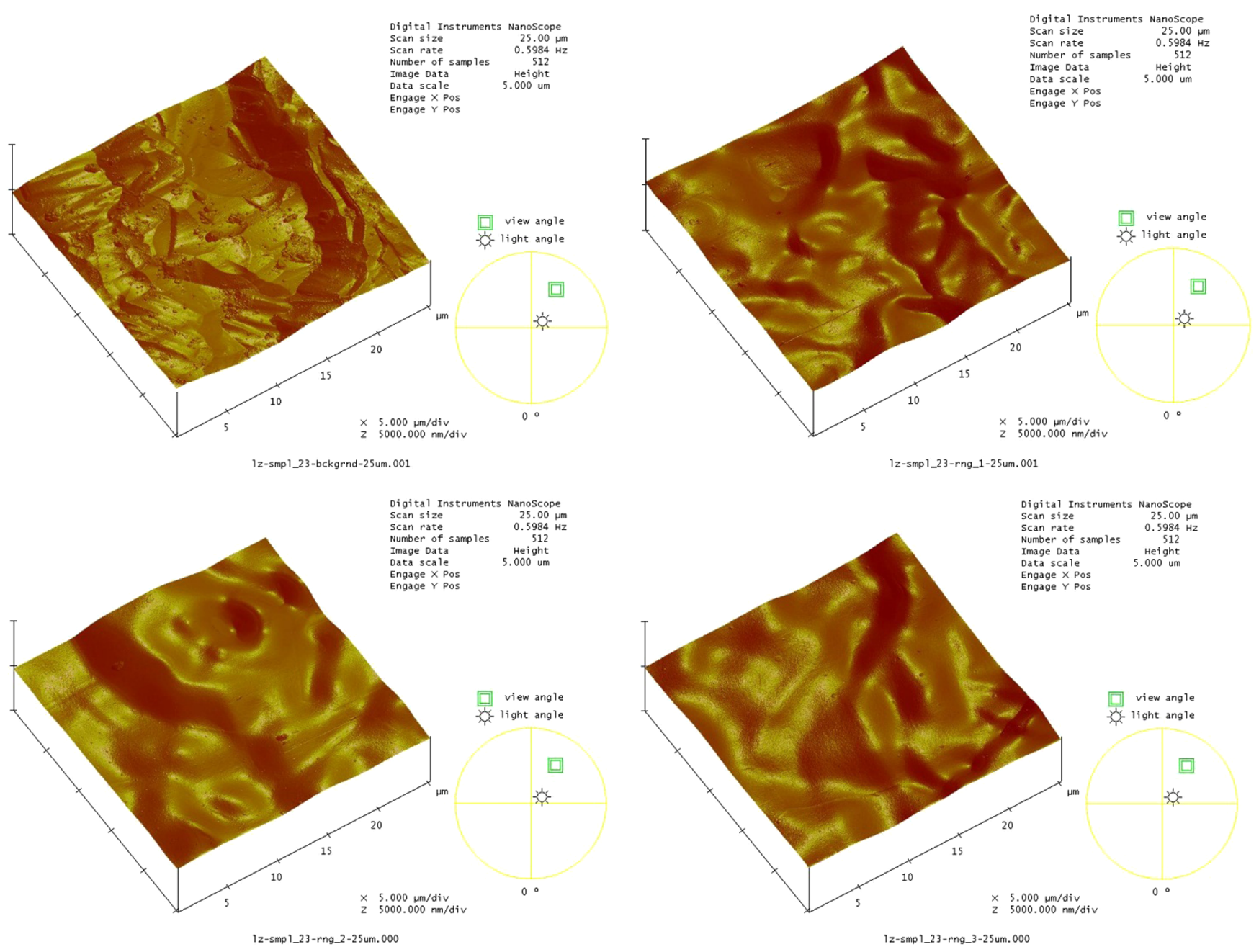

FIG. 7. AFM image of niobium surface after laser treatment with constant fluence $\left(2.27 \mathrm{~J} / \mathrm{cm}^{2}\right)$ and pulse accumulations. Top left, untreated BCP surface; top right, 11 pulses; lower left, 23 pulses; lower right, 34 pulses. Scan area: $25 \mu \mathrm{m} \times 25 \mu \mathrm{m}$. Horizontal scale: $5 \mu \mathrm{m}$ per division. Vertical scale: $0.5 \mu \mathrm{m}$ per division.

characteristic straight line on the log-log plot of PSD. The polished surfaces show a concave curve where the PSD values at high frequencies are lower than the $\mathrm{BCP}$ curve [22]. Laser treatment at 0.68 and $0.90 \mathrm{~J} / \mathrm{cm}^{2}$ produced a smoothened surface, while $1.13 \mathrm{~J} / \mathrm{cm}^{2}$ laser treatment produced a surface having critical topographic features similar to the starting BCP surface. We would relate the 0.68 and $0.90 \mathrm{~J} / \mathrm{cm}^{2}$ case to SMM, while the $1.13 \mathrm{~J} / \mathrm{cm}^{2}$ case shows more evidence of SOM. We have reported similar changes for $\mathrm{BCP}$ surfaces subjected to $\mathrm{EP}$ [23-26].

The next figures view the effect of varying pulse accumulation at constant fluence. The number of pulses overlapped (accumulated) within one pulse width (one beam spot diameter) ranged from 11 to 360 . The surface was slightly over melted around 38 pulses and the surface became damaged for pulse accumulations much greater than 38 pulses. For 23 pulses accumulation, the surface was polished. For 11 pulses accumulation, a

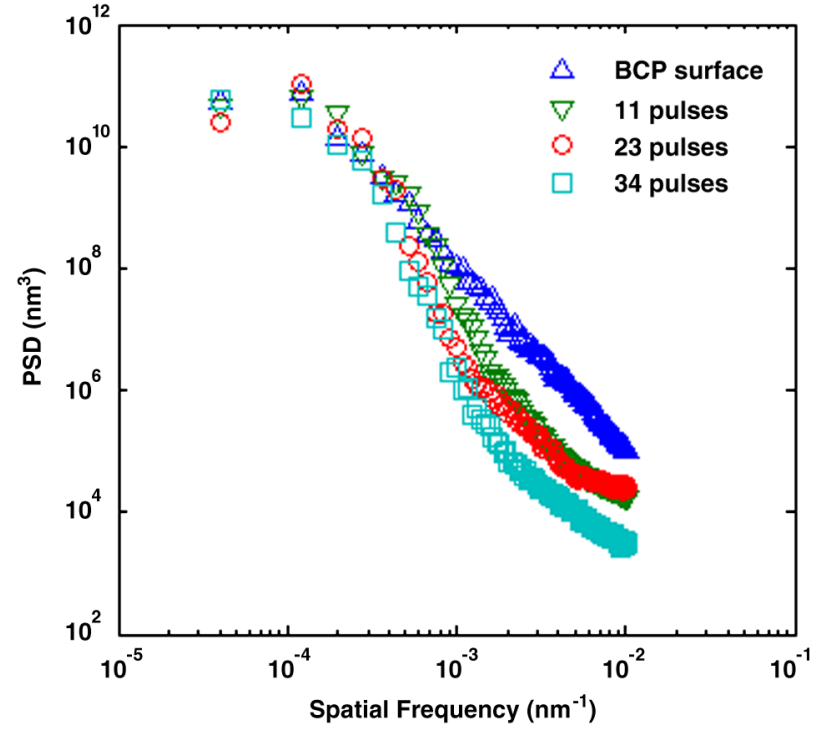

FIG. 8. PSD of AFM data corresponding to Fig. 7. 


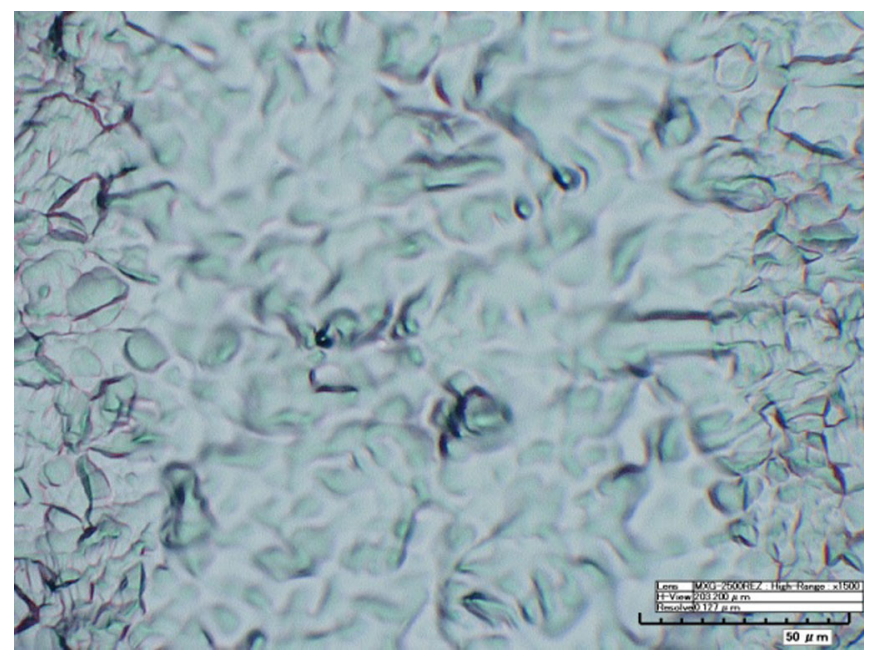

FIG. 9. Optical image of niobium surface after laser treatment with fluence $1.13 \mathrm{~J} / \mathrm{cm}^{2}, 34$ pulses accumulation, and five tracks with 0.6 overlapping ratio between two tracks. polishing effect was also achieved. Figure 7 shows the AFM images of niobium surface after laser treatment with different pulse accumulations (11, 23 and 34 pulses), at a constant fluence of $2.27 \mathrm{~J} / \mathrm{cm}^{2}$. Figure 8 is the corresponding PSD analysis of the images in Fig. 7. From 11 to 23 and then 34 pulses overlapped, the PSD shows steady improvement of surface smoothness.

In order to achieve laser treatment in a larger area, the laser beam was stepped along the radius while the target was rotating. The track of the laser beam spiraled from the edge towards the center of the disk, each circle overlapped with the next one at a chosen ratio. Using an optimized combination of fluence and pulse accumulation $\left(1.13 \mathrm{~J} / \mathrm{cm}^{2}, 34\right.$ pulses accumulated within one spot width), we were able to treat a larger area by overlapping tracks at different ratios. Figure 9 shows the optical image of niobium surface treated with 0.6 overlapping ratio. The removal of sharp edges on the surface is apparent.
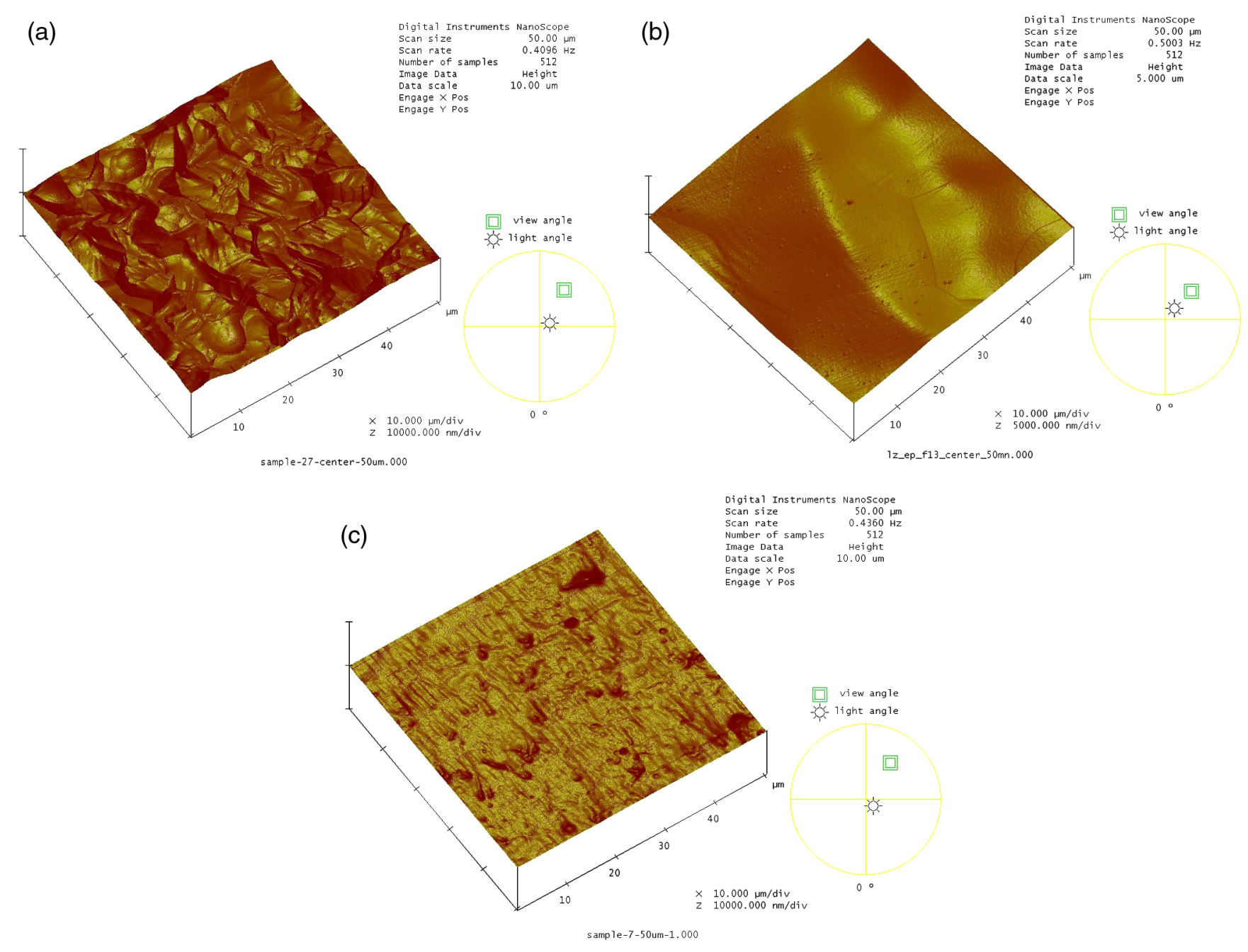

FIG. 10. AFM images of niobium surface after: (a) BCP; (b) EP; (c) centrifugal barrel polishing (CBP). Scan size was $50 \mu \mathrm{m} \times 50 \mu \mathrm{m}$. Horizontal scale: $10 \mu \mathrm{m}$ per division. Vertical scale: $10 \mu \mathrm{m}$ per division (a) and (c) and $5 \mu \mathrm{m}$ per division (b). 


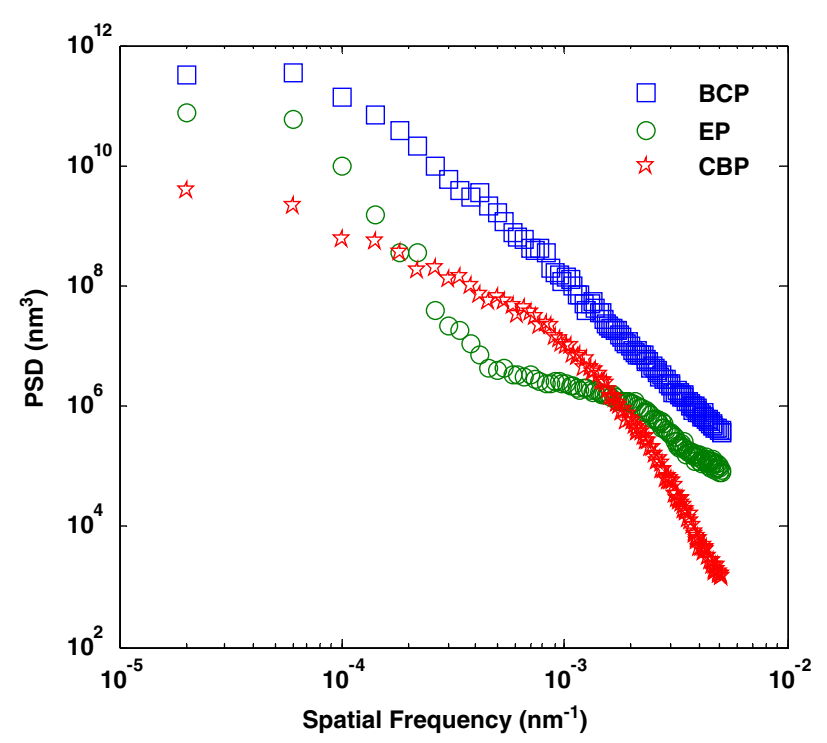

FIG. 11. PSD analyses of the BCP, EP, and CBP surfaces corresponding to the AFM images in Fig. 10.

\section{COMPARING LP WITH OTHER POLISHING METHODS}

We compared the topography of laser polishing (Figs. 6 and 8) with that of the other surface treatment methods (Figs. 10 and 11). Sample A was subjected to $100 \mu \mathrm{m}$ removal by 1:1:2 BCP ("heavy BCP") Sample B "EP" was first subjected to $100 \mu \mathrm{m}$ removal by $1: 1: 2 \mathrm{BCP}$ and then a further $40 \mu \mathrm{m}$ removal by electropolish, a heavy EP as previously described [27]. Sample C "CBP" is a typical CBP surface, prepared as described in Ref. [28]. The visible effect of EP on the PSD, even for as little as $5 \mu \mathrm{m}$ removal [29], is to eliminate the straight line (power law) profile associated with the sharp edges of $\mathrm{BCP}$, much as does laser polishing.

Figure 11 presents the PSD analysis of the surfaces in Fig. 10 in one plot for comparison. The BCP surface (square marker) shows almost a straight line going down at a slope of about $45^{\circ}$ from $6 \times 10^{-5} \mathrm{~nm}^{-1}$ to $5 \times 10^{-3} \mathrm{~nm}^{-1}$. The EP surface (circle marker) shows a recession in the middle of the $\mathrm{BCP}$ downward $45^{\circ}$ line from $6 \times 10^{-5} \mathrm{~nm}^{-1}$ to $3 \times 10^{-3} \mathrm{~nm}^{-1}$. We interpret this as the features in this frequency range were significantly reduced on EP surface comparing to BCP surface, with a turning point around $5 \times 10^{-4} \mathrm{~nm}^{-1}$ where the reduction slowed down and started to plateau out towards higher frequency. The CBP surface (star marker) curve shows lower PSD than the other curves over most of the frequency range except the slight hump around $10^{-3} \mathrm{~nm}^{-1}$. Laser polished (LP) surfaces (Figs. 6 and 8) show concave curvature having a lower PSD value than BCP line being from $4 \times 10^{-4} \mathrm{~nm}^{-1}$ to $10^{-2} \mathrm{~nm}^{-1}$. We interpret this as the high frequency features (sharp edges) on BCP surface were reduced, while the lower frequency features of the background $\mathrm{BCP}$ surface stayed unchanged. The LP curve overlapped the EP curve towards the high frequency end and at the low frequency limit, meaning the features within this range were similar on these two types of surfaces.

\section{CONCLUSIONS}

Laser polishing of niobium was achieved by controlling fluence and pulse accumulation. Images and PSD analysis showed that the best polishing result was with a fluence of $0.90-1.13 \mathrm{~J} / \mathrm{cm}^{2}$. In this fluence range, 34 pulses overlapped within one spot width provided the smoothest surface.

Larger area polishing was achieved by rastering laser tracks next to each other with appropriate $(\sim 60 \%)$ overlapping ratio.

\section{ACKNOWLEDGMENTS}

We gratefully acknowledge the support of the Office of High Energy Physics of the U.S. Department of Energy under Grant No. SC0007907 to the College of William \& Mary. The experiments were conducted at Jefferson Lab. This work was partly authored by Jefferson Science Associates, LLC under U.S. DOE Contract No. DEAC05-06OR23177. Thanks to staff at the College of William and Mary characterization lab, especially Olga Trofimova for the AFM images. Thanks to Chen Xu for the PSD program, and Ari Palczewski for helping with the CBP samples. We thank Professor Peter Schaaf of Gottingen for providing the computer code used for the model calculations.

[1] C. E. Reece and G. Ciovati, Rev. Accel. Sci. Techol. 05, 285 (2012).

[2] P. Kneisel, in Proceedings of the First Workshop on RF Superconductivity, Karlsruhe, Germany, 1980 (JACoW, Karlsruhe, Germany, 1980), pp. 27-40.

[3] H. Padamsee, J. Knobloch, and T. Hayes, RF Superconductivity for Accelerators, 2nd ed. (Wiley VCH, New York, 2008).

[4] H. Tian, S. G. Corcoran, C. E. Reece, and M. J. Kelley, J. Electrochem. Soc. 155, D563 (2008).

[5] C. Cooper, K. Saito, B. Bullock, S. Joshi, and A. Palczewski, in Proceedings of the 15th Workshop on RF Superconductivity, Chicago, IL, 2011 (JACoW, Chicago, IL, 2011), pp. 571-575.

[6] A. D. Palczewski, H. Tian, and R. L. Geng, in Proceedings of the 3rd International Particle Accelerator Conference, New Orleans, LA, 2012 (IEEE, Piscataway, NJ, 2012), pp. 2435-2437.

[7] V. Palmieri, G. Mondin, V. Rampazzo, D. Rizzetto, V. Rupp, S. Stivanello, S. Deambrosis, and A. A. Rossi, in Proceedings of the 14th Workshop on RF Superconductivity, Berlin, Germany, 2009 (JACoW, Berlin, Germany, 2009), pp. 463-465. 
[8] A. I. Wixtrom, J. E. Buhler, C. E. Reece, and T. M. Abdel-Fattah, J. Electrochem. Soc. 160, E22 (2013).

[9] M. Inman, T. Hall, E. J. Taylor, C. E. Reece, and O. Trofimova, in Proceedings of the 15th Workshop on RF Superconductivity, Chicago, IL, 2011 (JACoW, Chicago, IL, 2011), pp. 377-381.

[10] E. Taylor, in Proceedings of the 16th International Conference on RF Superconductivity, Paris, France, 2013 (JACoW, Paris, France, 2013), pp. 534-537.

[11] M. Inman, E. J. Taylor, and T. D. Hall, J. Electrochem. Soc. 160, E94 (2013).

[12] M. Rašković, J. Upadhyay, S. Popović, L. Vušković, A.-M. Valente-Feliciano, and L. Phillips, in Proceedings of the 11th European Particle Accelerator Conference, Genoa, 2008 (EPS-AG, Genoa, Italy, 2008), pp. 928-930.

[13] J. Upadhyay, M. Rašković, L. Vušković, S. Popović, L. Phillips, and A.-M. Valente-Feliciano, in Proceedings of the 14th Workshop on RF Superconductivity, Berlin, Germany, 2009 (JACoW, Berlin, Germany, 2009), pp. 427-430.

[14] S. Singaravelu, J. M. Klopf, C. Xu, G. Krafft, and M. J. Kelley, J. Vac. Sci. Technol. B 30, 051806 (2012).

[15] L. Zhao, J. M. Klopf, C. E. Reece, and M. J. Kelley, in Proceedings of the 4th International Particle Accelerator Conference, IPAC-2013, Shanghai, China, 2013 (JACoW, Shanghai, China, 2013), p. 2498.

[16] J. A. Ramos, D. L. Bourell, and J. J. Beaman, Mater. Res. Soc. Symp. Proc. 758, LL1.9 (2002).

[17] E. Ukar, A. Lamikiz, L. N. Lopez de Lacalle, D. del Pozo, and J. L. Arana, Intl. J. Mach. Tools Manufacture 50, 115 (2010).
[18] T. L. Perry, D. Werschmoeller, N. A. Duffie, X. Li, and F. E. Pfefferkorn, ASME J. Manuf. Sci. Eng. 131, 021002 (2009).

[19] T. A. Mai, and G. C. Lim, J. Laser Appl. 16, 221 (2004).

[20] M. Bereznai, I. Pelsoczi, Z. Toth, K. Turzo, M. Radnai, Z. Bor, and A. Fazekas, Biomaterials 24, 4197 (2003).

[21] F. Vega, N. Lupon, J. A. Cebrian, and F. Laguarta, Opt. Eng. (Bellingham, Wash.) 37, 272 (1998).

[22] S. Singaravelu, J. M. Klopf, G. Krafft, and M. J. Kelley, J. Vac. Sci. Technol. B 29, 061803 (2011).

[23] H. Tian, C. E. Reece, M. J. Kelley, S. Wang, L. Plucinski, K. E. Smith, and M. M. Nowell, Appl. Surf. Sci. 253, 1236 (2006).

[24] C. Xu, H. Tian, C. E. Reece, and Michael J. Kelley, Phys. Rev. ST Accel. Beams 15, 043502 (2012).

[25] C. Xu, H. Tian, C. E. Reece, and M. J. Kelley, Phys. Rev. ST Accel. Beams 14, 123501 (2011).

[26] H. Tian, G. Ribeill, C. Xu, C. E. Reece, and M. J. Kelley, Appl. Surf. Sci. 257, 4781 (2011).

[27] L. Zhao, M. Kelley, and C. Reece, in Proceedings of the 24th Particle Accelerator Conference, PAC-2011, New York, 2011 (IEEE, New York, 2011), pp. 1038-1040.

[28] A. D. Palczewski, G. Ciovati, R-L. Geng, and Y. Li, in Proceedings of the 16th International Conference on RF Superconductivity, Paris, France, 2013 (JACoW, Paris, France, 2013), pp. 573-576.

[29] L. Zhao, J. M. Klopf, C. E. Reece, and M. J. Kelley, in Proceedings of the 16th International Conference on RF Superconductivity, Paris, France, 2013 (JACoW, Paris, France, 2013), pp. 587-589. 